\title{
Thermodynamic and morphological behavior of block copolymer blends with thermal polymer additives.
}

\author{
Daniel F. Sunday $^{1{ }^{* *}}$, Adam F. Hannon ${ }^{1 \neq}$, Summer Tein ${ }^{2}$, R. Joseph Kline ${ }^{1}$ \\ 1. Materials Science and Engineering Division, National Institute of Standards and Technology, \\ 100 Bureau Drive, Gaithersburg, MD 20899 \\ 2. McKetta Department of Chemical Engineering, University of Texas, Austin TX
}

\section{Supplemental Information}

\section{S1 Simulation Testing/Calibration:}

To ensure the simulations were accurate, a set of 1D simulations using B homopolymer was performed with different $N_{\mathrm{B}}^{\mathrm{H}}$ values relative to the diblock $N_{\mathrm{AB}}$. The parameter $\alpha=\frac{N_{\mathrm{B}}^{\mathrm{H}}}{N_{\mathrm{AB}}}$ is used to distinguish different molecular weight ratios of homopolymer to diblock. This parameter was chosen rather than the $\eta$ used in the main text to correspond to the same parameter used by Matsen et $\mathrm{al}^{1}$. If the segment volume of both $\mathrm{A}$ and $\mathrm{B}$ species are the same, $\alpha=\eta f$, otherwise $\alpha=\eta N_{\mathrm{B}}^{\mathrm{BCP}} / N_{\mathrm{AB}} . N_{\mathrm{AB}}$ was fixed as 200 and $N_{\mathrm{B}}^{\mathrm{H}}$ was varied with values $10,20,50,100$, and 200 corresponding to $\alpha$ values of $1 / 20,1 / 10,1 / 4,1 / 2$, and 1 , respectively. $f_{\mathrm{B}}^{\mathrm{H}}$ was varied from 0.01 to 0.33 in steps of 0.04 and a run using pure $N_{\mathrm{AB}}=200$ was also performed for a baseline reference. In order to extract $L$, the natural periodicity of the copolymer system under the given blending conditions, simulations were performed assigning an incremental length scale in units of $R_{\mathrm{g}}$ to the simulation box going from $\approx 1.65 R_{\mathrm{g}}$ to $\approx 6.6 R_{\mathrm{g}}\left(L_{0}\right.$ for the bulk diblock copolymer is expected to have period $\approx 3$ to $4 R_{\mathrm{g}}$ from strong segregation theory) ${ }^{2} . R_{\mathrm{g}}$ is calculated using the relation $R_{\mathrm{g}}=b N^{1 / 2} / 6^{1 / 2}$, where $b$ is the Kuhn segment length of the monomer which is assumed to be 0.25 a.u. in this study for all three monomers. From the simulation results using incremental box sizes, the free energy as a function of box size was plotted along with the kind of morphology that was observed at those conditions. An example of these plots with typical kinds of observed morphologies for different blending conditions is shown in Figure S1. In 
general for all 1D simulations, as long as the copolymer phase segregated, a primary free energy curve was observed corresponding to a unit cell 1D lamellar morphology. To extract $L$ from these results, a strain dominated relationship for the free energy was fit to the free energy curve for those data points that corresponded to the 1D lamellar morphology using the equation $\Delta F_{\text {fit }} / k T=\Gamma \Lambda^{2}+\beta / \Lambda-\epsilon$ and the constants $\Gamma, \beta$, and $\epsilon$ fit using a CMAES optimization ${ }^{3-5}$ yielding the final $L=(\beta /(2 \Gamma))^{1 / 3}$. Here $\Lambda$ is the assigned unit cell length.
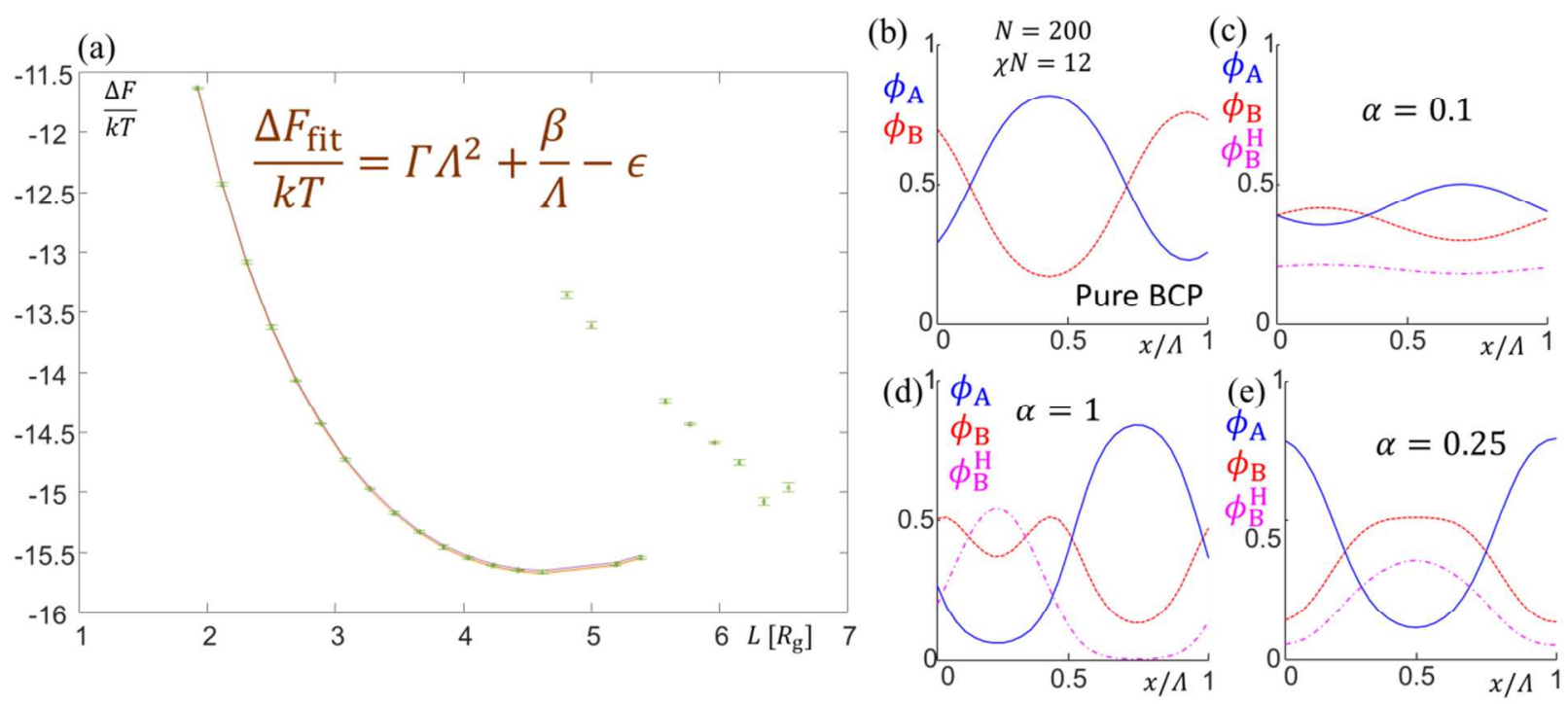

Figure S1: (a) Plot of the normalized free energy difference $\Delta F / k T$ from a disordered state $(\Delta F / k T=0$ for at the disordered state with negative energies indicating ordering has occurred) for the case of $N_{\mathrm{AB}}=500$ with $f_{\mathrm{C}}=0.01$ for $\chi_{\mathrm{BC}}=-0.01$ and $N_{\mathrm{C}}=54$. Green dots are calculated values from 1D SCFT simulations with standard deviation as bars. The brown lines are fits to the data using the strain dominated free energy function $\Delta F_{\text {fit }} / k T$ for the data that corresponds to a single repeating of the copolymer 1D lamellae structure. Other data was double 1D lamellae within a single unit cell (which was a local metastable structure in some cases where the single 1D lamellae structure would clearly be lower in free energy). (b-e) $1 \mathrm{D}$ normalized density $\phi$ plots versus normalized unit cell position $x / \Lambda$ for various conditions of $\mathrm{AB}$ diblocks blended with $f_{\mathrm{B}}^{\mathrm{H}}=0.21$ A homopolymer where applicable. Blue curves correspond to the A block densities, red dashed curves to the B block densities, and magenta dotted curves to the B homopolymer densities. Conditions were for an $N_{\mathrm{AB}}=200$ and $\chi N=12$ interaction parameter. (b) Density plots for the pure block copolymer for reference. The B block has a slightly lower peak than the A block as the volume fraction $f_{\mathrm{B}}=0.45<0.5$. (c) $\alpha=0.1$ case where the homopolymer addition has started to induce homogenational disordering of the system. The effective $L / L_{0} \cong 0.85<1$. (d) $\alpha=1$ case where the homopolymer addition has started to induce macrophase separation in the system as indicated by the double peaks of the B block with the homopolymer residing inside the center of the B block region. The effective $L / L_{0} \cong 1.40>1$. (e) $\alpha=0.25$ case where the homopolymer simply overlaps with the B block density. The effective $L / L_{0} \cong 1.05$.

These $L$ values in the a.u. of the simulation were all calculated in similar fashion and then normalized by $b N_{\mathrm{AB}}^{1 / 2}$ for comparison with data previously found by Matsen ${ }^{1}$. As seen in Figure $\mathrm{S} 2$, the same trends of going from a negative slope with increasing $f_{\mathrm{B}}^{\mathrm{H}}$ to a positive slope by 
increasing $\alpha$ was observed in both the previous study and the current set of simulations. Except for the case of $\alpha=1$, similar values of $\alpha$ in both studies gave similar slopes. Discrepancies between the studies may be from the fact the previous study did not specify the exact $N_{\mathrm{AB}}$ that was used, only $\chi N=12$ which was matched. There should not be a huge effect of $N_{\mathrm{AB}}$ as another trial run using $N_{\mathrm{AB}}=100$ was tested yielding similar behavior. Regardless, the fact the overall trend is the same and most $\alpha$ values quantitatively matched gave credence to continue with the parameters as optimized.

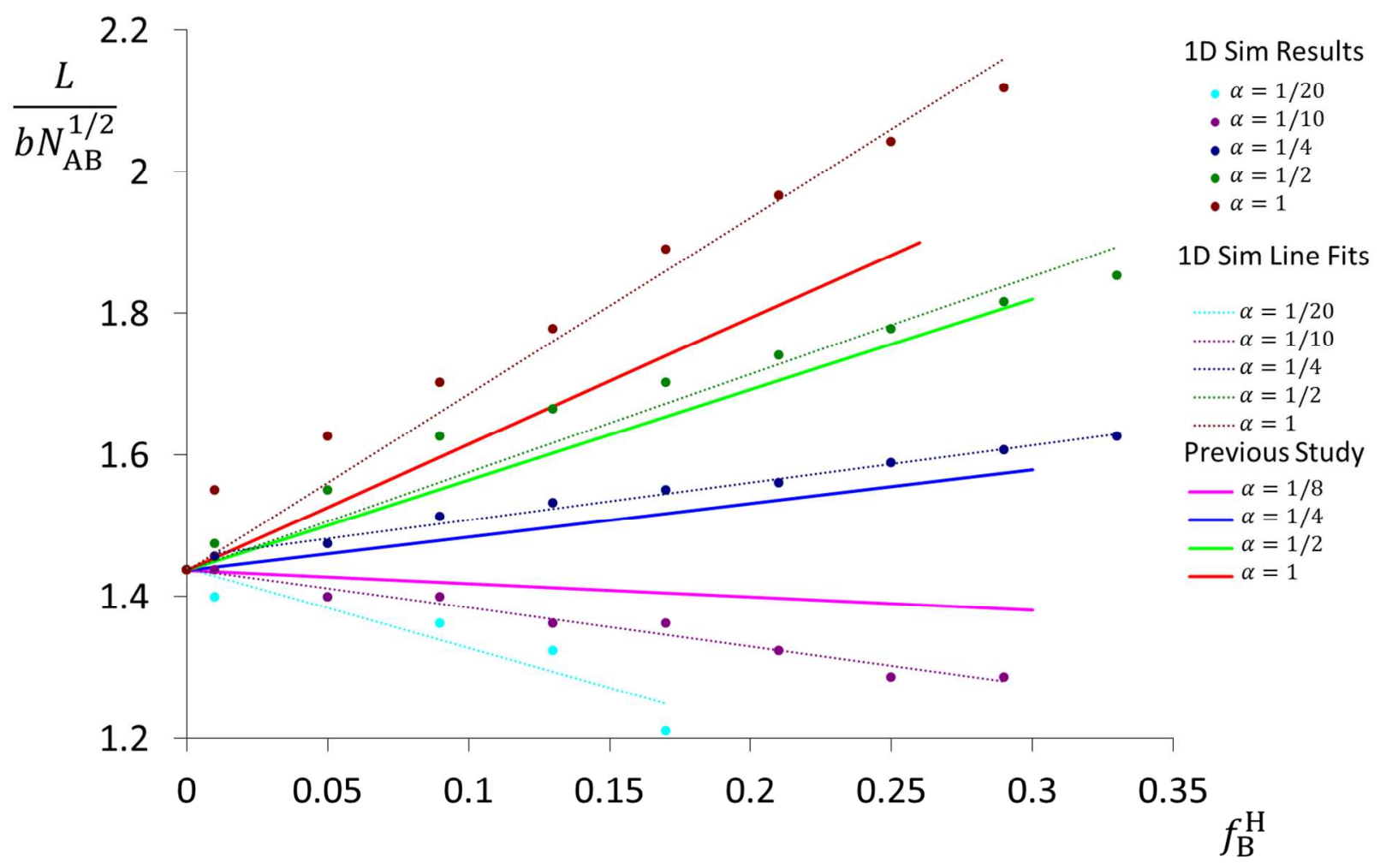

Figure S2: Plot of calculated normalized $L$ values (dots) as a function of incorporated B homopolymer fraction $f_{\mathrm{B}}^{\mathrm{H}}$ plotted alongside data previously obtained by Matsen ${ }^{1}$ (solid lines). Here $N_{\mathrm{AB}}=200$ and $\chi N=12$ for the simulated results. Data points have line fits (dashed lines) added to guide the eye that are forced to fit through the point $f_{\mathrm{B}}^{\mathrm{H}}=0 \rightarrow L / b N^{1 / 2} \cong 1.44$. Color coding is such that cyan is $\alpha=1 / 20$, purple is $\alpha=1 / 10$, navy is $\alpha=1 / 4$, forest green is $\alpha=1 / 2$, and crimson is $\alpha=1$ for the simulated results. For the previous study data lines, magenta is $\alpha=1 / 8$, blue is $\alpha=1 / 4$, green is $\alpha=1 / 2$, and red is $\alpha=1$.

\section{S2 Simulation Results:}

To examine the dependence of $L / L_{0}$ as a function of $f_{\mathrm{B}}^{\mathrm{H}}$ or $f_{\mathrm{C}}$, an initial set of $1 \mathrm{D}$ simulations looking at how $L / L_{0}$ varied with $\mathrm{B}$ homopolymer incorporation for the highest molecular weight $N_{\mathrm{AB}}=811$ was performed and compared with the experimental data. Both $N_{\mathrm{B}}^{\mathrm{H}}=65$ and 175 were performed corresponding to $\alpha$ values of 0.080 and 0.216 . The results of 
this comparison are shown in Figure S3. As seen in the figure, the three experimental points fall very close to the simulated data for the $N_{\mathrm{B}}^{\mathrm{H}}=175$ case, but not as close to the data for the $N_{\mathrm{B}}^{\mathrm{H}}=65$ case which corresponds better to the actual molecular weight of the system. All simulations for testing the PVPH incorporation tested both the expected degree of polymerization of the PVPH homopolymer of $N_{\mathrm{C}}=54$ as well as a larger value of $N_{\mathrm{C}}=200$.

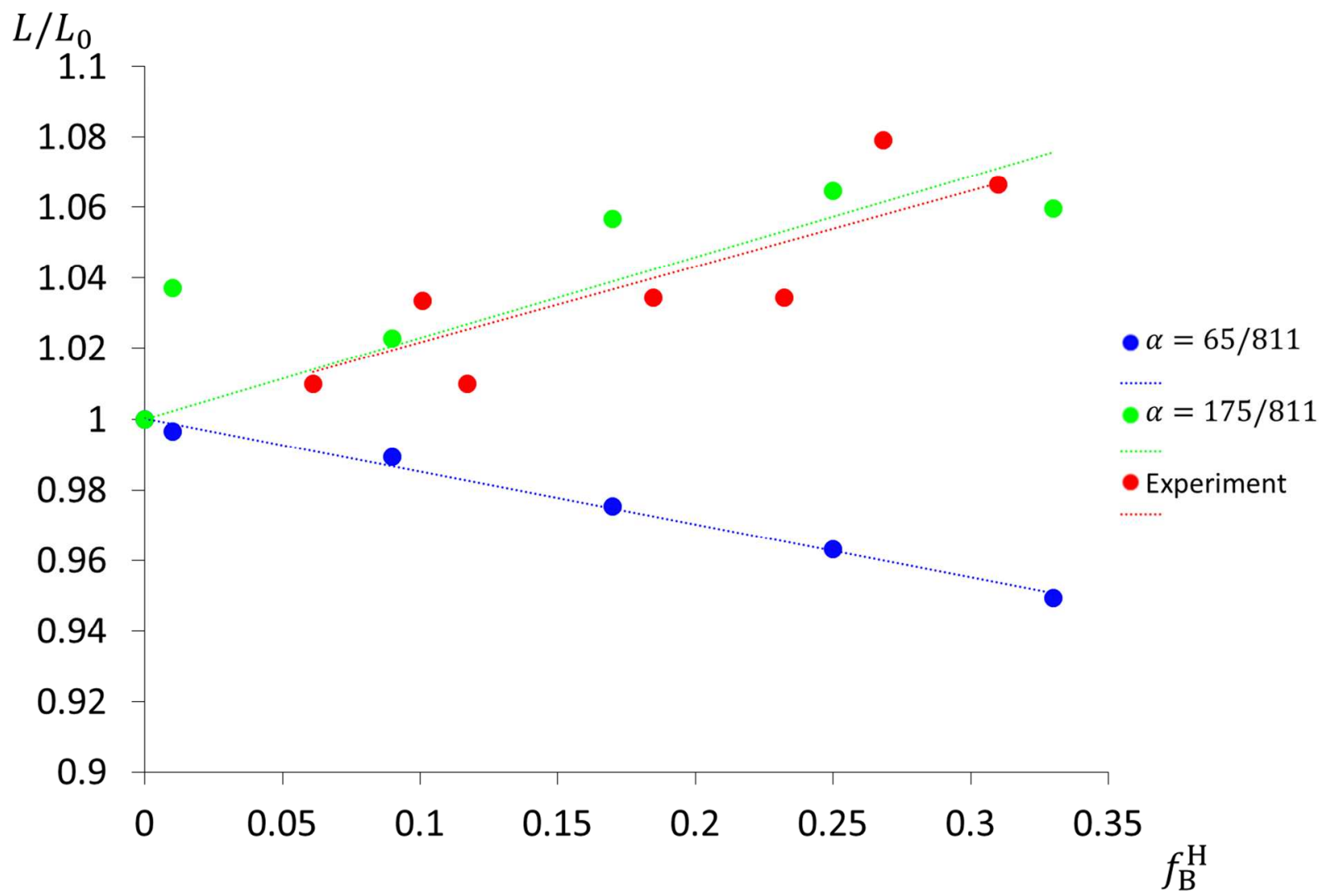

Figure S3: Plots of normalized domain spacing $L / L_{0}$ versus incorporated homopolymer volume fraction $f_{\mathrm{B}}^{\mathrm{H}}$. Dots are calculated data from free energy curve fits and dash lines are linear fits to the data forced to fit $f_{\mathrm{B}}^{\mathrm{H}}=0 \rightarrow L / L_{0}=1$ to guide the eyes. Blue data is for $\alpha=65 / 811 \cong 0.080$ and uses $N_{\mathrm{B}}^{\mathrm{H}}=65$ which is expected degree of polymerization for the homopolymer in the system based on the provided molecular weight. Green data is for $\alpha=175 / 811 \cong 0.216$ using a larger $N_{\mathrm{B}}^{\mathrm{H}}=175$. Red data is what was experimentally found using SAXS measurements.

Thirty-two total sets of parameter combinations were tested over a range of $f_{\mathrm{C}}=$ 0.01 to 0.35 in steps of 0.02 to gauge which combination of $N_{\mathrm{C}}$ and $\chi_{\mathrm{BC}}$ best matched the experimental data found using SAXS and SANS. Figure S4 shows the simulation results of $L / L_{0}$ versus $f_{\mathrm{C}}$ for the $N_{\mathrm{C}}=54$ case for all $\chi_{\mathrm{BC}}$ tested and Figure S5 shows the results for the $N_{\mathrm{C}}=$ 200 case. As seen in the figures for the $N_{\mathrm{C}}=54$ case, the increase of $L / L_{0}$ with $f_{\mathrm{C}}$ for the higher molecular weights $\left(N_{\mathrm{AB}}=500\right.$ and 811$)$ did not vary much with $\chi_{\mathrm{BC}}$ except for very larger $f_{\mathrm{C}}$ in 
which case the less negative $\chi_{\mathrm{BC}}$ values (i.e. closer to 0 ) tended to give larger increases in periodicity. On the other hand, the lower molecular weights $\left(N_{\mathrm{AB}}=183\right.$ and 196) had more distinct $L / L_{0}$ versus $f_{\mathrm{C}}$ curves with the less negative $\chi_{\mathrm{BC}}$ values having larger $L / L_{0}$ values as $f_{\mathrm{C}}$ increased. As $\chi_{\mathrm{BC}}$ became more negative for the lower molecular weights, the decrease in $L / L_{0}$ at larger $f_{\mathrm{C}}$ became less implying increasing the attractive strength of the $\mathrm{B}$ and $\mathrm{C}$ species has a diminishing effect on shrinking the effective periodicity relative to the $\chi_{\mathrm{BC}}=0$ condition. The difference in the observed decreases in $L / L_{0}$ with more negative $\chi_{\mathrm{BC}}$ values for the lower molecular weights and negligible changes for the higher molecular weights are likely due to the fact the higher molecular weights phase segregate naturally before any $\mathrm{C}$ homopolymer is added to the system while the lower molecular weights require $f_{\mathrm{C}} \cong>0.05$ before phase segregation is induced. The strength of the attractive $\mathrm{BC}$ interactions has a larger impact on this chain stretching for the initially disordered system than in the case of the higher molecular weights where the chain stretching is dominated by the higher $\mathrm{AB}$ repulsive interactions from the longer $\mathrm{AB}$ chains. As a general trend that was observed, decreasing the $\mathrm{AB}$ copolymer molecular weight increased the corresponding $L / L_{0}$ values (meaning shorter chains were stretched relatively more than longer chains with added $\mathrm{C}$ homopolymer). 


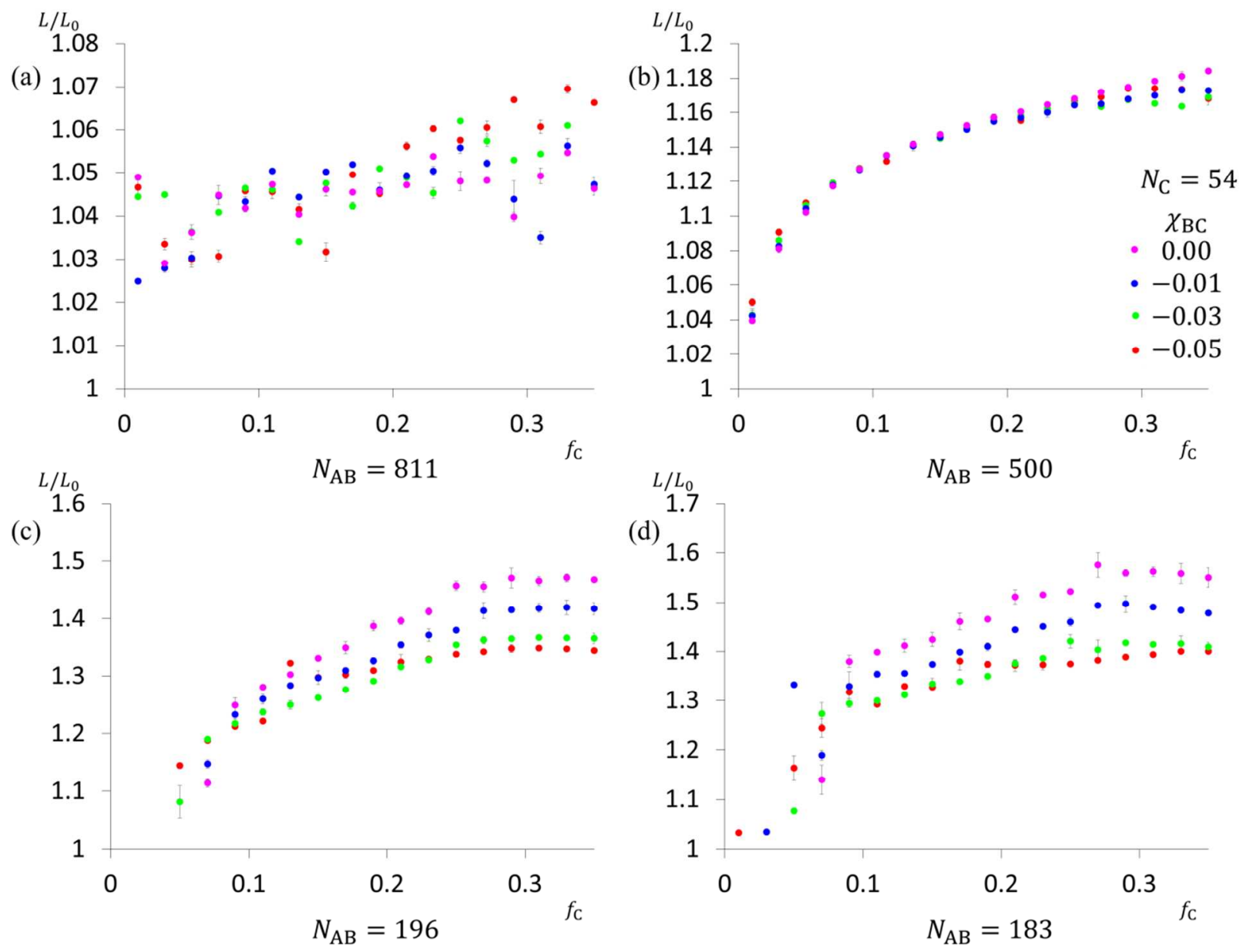

Figure S4: Plots of the normalized periodicity $L / L_{0}$ versus increasing $\mathrm{C}$ homopolymer fraction $f_{\mathrm{C}}$ from $1 D$ SCFT simulations. Here the $\mathrm{C}$ homopolymer has degree of polymerization $N_{\mathrm{C}}=54$. Dot colors are magenta, blue, green, and red for $\chi_{\mathrm{BC}}=0,-0.01,-0.03$, and -0.05 , respectively. Uncertainty bars are standard uncertainty from the free energy calculations. (a) $N_{\mathrm{AB}}=811$. (b) $N_{\mathrm{AB}}=500$. (c) $N_{\mathrm{AB}}=196$. (d) $N_{\mathrm{AB}}=183$.

For the higher molecular weight $\mathrm{C}$ homopolymer with $N_{\mathrm{C}}=200$, a more clear trend of decreasing $L / L_{0}$ with a more negative $\chi_{\mathrm{BC}}$ parameter was observed for all $N_{\mathrm{AB}}$ values. Since $N_{\mathrm{C}}$ is larger, the effects of the $\mathrm{BC}$ interactions become comparable to the $\mathrm{AB}$ interactions and thus a change in $L / L_{0}$ results from this effect. Another observation is that the relative $L / L_{0}$ for a given $N_{\mathrm{AB}}$ are larger than the corresponding values for the $N_{\mathrm{C}}=54$ cases as one would expect from similar trends in homopolymer blending where one species is the same as one of the blocks. For the lower $N_{\mathrm{AB}}$ blocks, the transition from the disordered state has a much larger $L / L_{0}$ value observed $>2$ in some cases and decreases initially with increasing $f_{\mathrm{C}}$ before again increasing. This behavior is likely due to the disordered homogeneous state transitioning to disordered micelles rather than normal lamellae (the two phases are almost indistinguishable in $1 \mathrm{D}$ except 
for the free energy curves having different minimum locations). Thus the free energy fits for those states are likely from disordered 1D micelles rather than 1D lamellae phases.


Figure S5: Plots of the normalized periodicity $L / L_{0}$ versus increasing $\mathrm{C}$ homopolymer fraction $f_{\mathrm{C}}$ from $1 \mathrm{D}$ SCFT simulations. Here the $\mathrm{C}$ homopolymer has degree of polymerization $N_{\mathrm{C}}=200$. Dot colors are magenta, blue, green, and red for $\chi_{\mathrm{BC}}=0,-0.01,-0.03$, and -0.05 , respectively. Uncertainty bars are standard uncertainty from the free energy calculations. (a) $N_{\mathrm{AB}}=811$. (b) $N_{\mathrm{AB}}=500$. (c) $N_{\mathrm{AB}}=196$. (d) $N_{\mathrm{AB}}=183$.

Comparing the various simulation results with the experiment, the parameter combination of $N_{\mathrm{C}}=54$ and $\chi_{\mathrm{BC}}=-0.05$ appeared to match well with the experimental results (taking into account both general trends of $L / L_{0}$ versus $f_{\mathrm{C}}$ as well as the $3 \mathrm{D}$ phase space morphologies detailed in the main text). Comparison plots of the different $\chi_{\mathrm{BC}}=-0.05$ data with the various molecular weights are shown in Figure S6. For the higher $N_{\mathrm{AB}}$ cases with $N_{\mathrm{C}}=200$, the $N_{\mathrm{AB}}$ data has much larger $L / L_{0}$ values than the corresponding simulation data except for large $f_{\mathrm{C}}$ values, but even then the corresponding experimental data falls between the two simulation curves. On the other hand, for the $N_{\mathrm{C}}=54$ cases, the low $f_{\mathrm{C}}$ simulation values fall right within 
the ranges of $L / L_{0}$ observed in the experiment. However, the large $f_{\mathrm{C}}$ values $>0.2$ appear to start asymptotically leveling from the simulation while they appeared to continue to increase in the experiment.

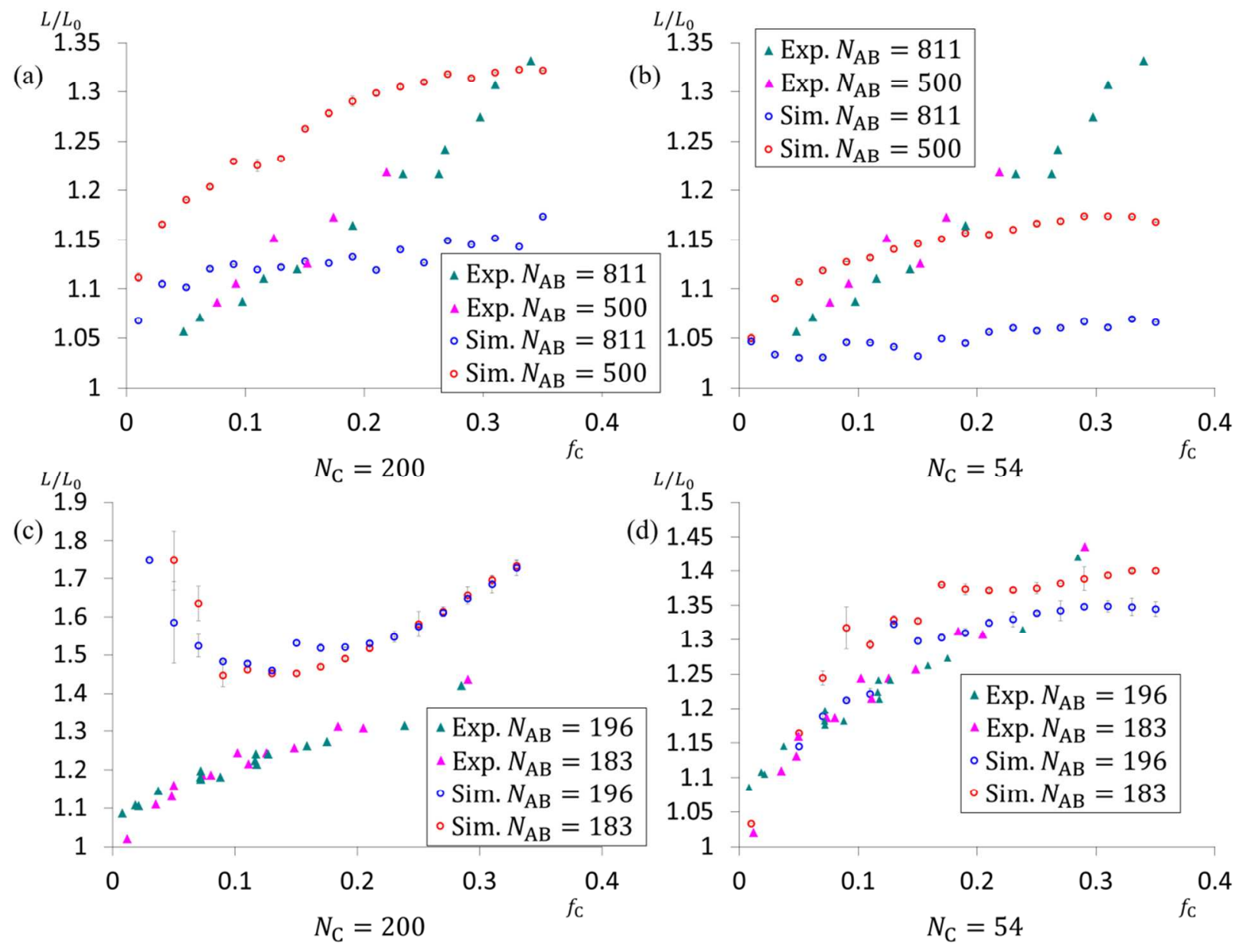

Figure S6: Plots of the normalized periodicity $L / L_{0}$ versus increasing $\mathrm{C}$ homopolymer fraction $f_{\mathrm{C}}$ from 1D SCFT simulations compared with the experimental data. Uncertainty bars are standard uncertainty from the free energy calculations. (a-b) Marker colors are teal or blue and magenta or red for $N_{\mathrm{AB}}=$ 811 and 500, respectively, with triangles being experiment data and circles being simulation results. (a) $N_{\mathrm{C}}=200$. (b) $N_{\mathrm{C}}=54$. (c-d) Marker colors are teal or blue and magenta or red for $N_{\mathrm{AB}}=196$ and 183, respectively, with triangles being experiment data and circles being simulation results. (c) $N_{\mathrm{C}}=200$. (d) $N_{\mathrm{C}}=54$.

For the lower molecular weight cases with $N_{\mathrm{C}}=200$, the simulation data significantly overestimates the observed experimental $L / L_{0}$ values. On the other hand, the $N_{\mathrm{C}}=54$ results match very well with the observed experimental values with similar trends and ranges of $L / L_{0}$ values. 


\section{S3 Normalization Extrapolation:}

Since the $N_{\mathrm{AB}}=183$ and 196 pure AB diblocks are below their ODT $\chi N$, extrapolation of the values for $L_{0}$ at $f_{\mathrm{C}}=0$ was necessary to compare with the experimental results. Based on the observed functional shape of $L$ with increasing $f_{\mathrm{C}}$ for the $N_{\mathrm{C}}^{\mathrm{H}}=54$ appearing exponential in nature, a fit equation of $L=L_{\text {Sat }}+\left(L_{0}-L_{\text {Sat }}\right) e^{-\zeta f_{\mathrm{C}}}$ was used to find what the normalization of $L_{0}$ should be. As seen in this equation form, $L \rightarrow L_{0}$ as $f_{\mathrm{C}} \rightarrow 0$ and $L \rightarrow L_{\text {Sat }}$ for large $f_{\mathrm{C}}$, with $\zeta$ controlling the rate at which $L$ increases towards $L_{\text {Sat }}$ with increasing $f_{\mathrm{C}}$. A plot of these fits are shown for the case of $\chi_{\mathrm{BC}}=-0.05$ in Figure S7. From these fits, $L_{0}$ was found to be approximately $3.02 R_{\mathrm{g}}$ for both $N_{\mathrm{AB}}$ (at least within statistical uncertainty of the fits, intuitively the higher $N_{\mathrm{AB}}$ should also have a larger $L_{0}$, but they do not have the same $f_{\mathrm{B}}$ which may influence the values slightly). These values were thus used to normalize all the calculated $L$ values for their respective $N_{\mathrm{AB}}$ throughout the manuscript.

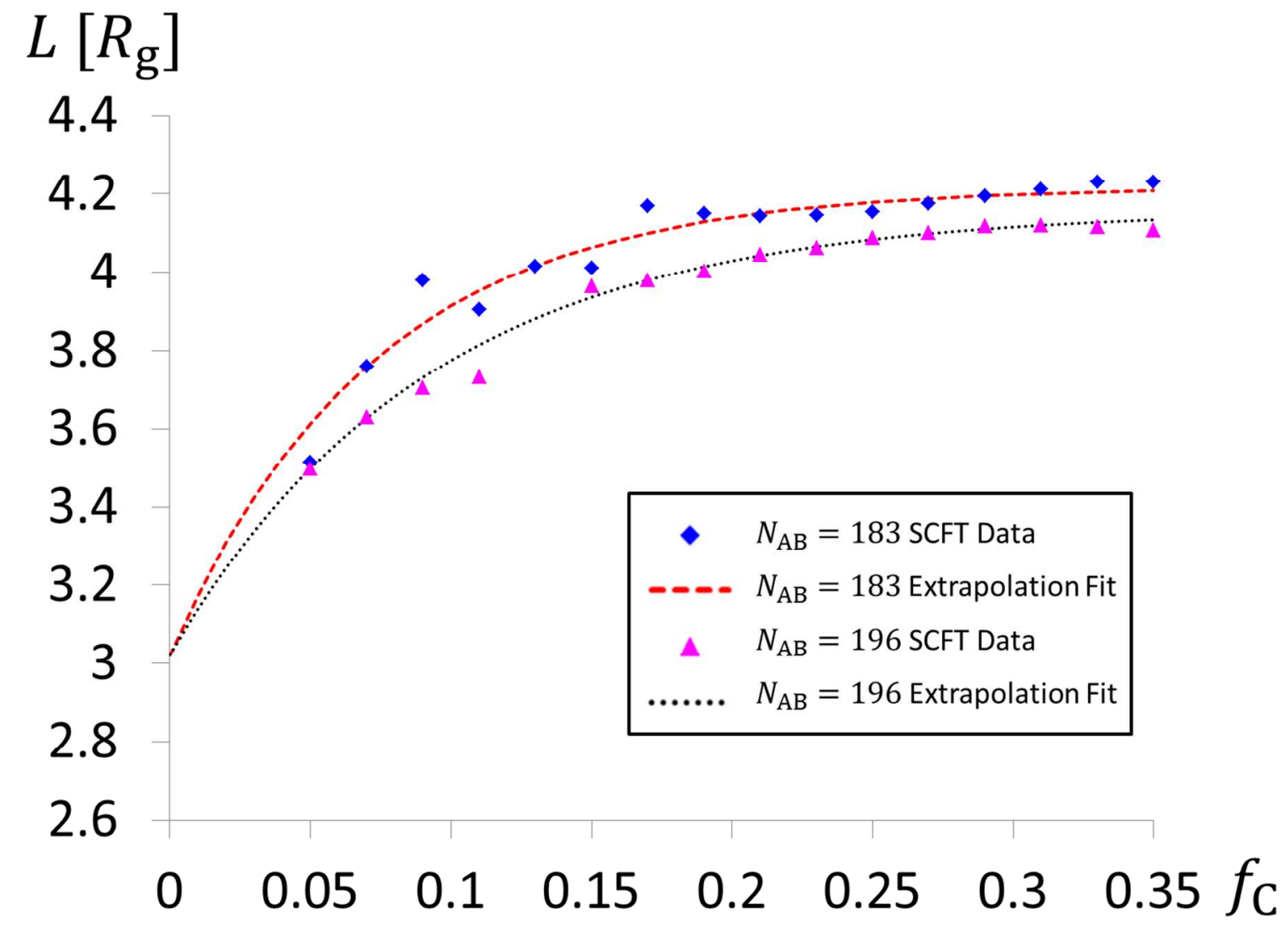


Figure S7: Plot of the extrapolation fits of the $\chi_{\mathrm{BC}}=-0.05$ SCFT data for both $N_{\mathrm{AB}}=183$ (fit as a red dashed line and data as blue diamonds) and $N_{\mathrm{AB}}=196$ (fit as a black dotted line and data as magenta triangles). Both fits approach a similar value of $3.02 R_{\mathrm{g}}$.

\section{S4 Rationalization of Phases Observed Only in Simulations:}

For the two lower $N_{\mathrm{AB}}$ diblocks in the simulations with $\mathrm{C}$ homopolymer, the observed phases with increasing $f_{\mathrm{C}}$ showed $\mathrm{B}$ and $\mathrm{C}$ rich spheres around $f_{\mathrm{C}}=0.05$ and $\mathrm{B}$ and $\mathrm{C}$ rich cylinders around $f_{\mathrm{C}}=0.09$. These phases were not observed experimentally, but can be rationalized from the simulations considering the standard mean field phase diagram for diblocks and the fact fluctuations were not taken into account in these system (complex Langevin dynamics was not used to arrive at the final phase). As $f_{\mathrm{C}}$ increases, the effective $\chi$ parameter between the $\mathrm{A}$ and $\mathrm{B}$ blocks will increase sharply initially as the $\mathrm{C}$ homopolymer blends with the B block. As additional $\mathrm{C}$ homopolymer is added to the system, the effective $\chi$ will continue to increase but not as sharply. While $\chi_{\text {eff }}$ increases, so too does the effective volume fraction of the B block. The effective fraction is given approximately as $f_{\text {eff }}=f_{\mathrm{B}} f_{\mathrm{AB}}+f_{\mathrm{C}}=f_{\mathrm{B}}\left(1-f_{\mathrm{C}}\right)+f_{\mathrm{C}}$ assuming complete miscibility between the $\mathrm{B}$ and $\mathrm{C}$ phases (thus the actual value with some partial miscibility of the $\mathrm{A}$ and $\mathrm{C}$ phases will result in a lower $f_{\text {eff }}$ ). Since $f_{\mathrm{B}}$ starts off less than 0.5 for both $N_{\mathrm{AB}}=183$ and $196\left(f_{\mathrm{B}}=0.42\right.$ and 0.47 , respectively $), f_{\text {eff }}$ does not reach 0.5 until about $f_{\mathrm{C}}=0.14$ for the $N_{\mathrm{AB}}=183$ case and $f_{\mathrm{C}}=0.6$ for the $N_{\mathrm{AB}}=196$ case. The actual $f_{\mathrm{C}}$ where the effective fractions cross will be higher because of partial miscibility of the $\mathrm{A}$ and $\mathrm{C}$ species. $\mathrm{B}$ and $\mathrm{C}$ rich cylinders were observed up to $f_{\mathrm{C}}=0.13$ and $f_{\mathrm{C}}=0.9$ for the 183 and 196 $N_{\mathrm{AB}}$ values, respectively, which roughly agrees to be around the upper limit of the $f_{\text {eff }}=0.5$ cutoff. Taking these ideas all together, in the mean field limit both low $N_{\mathrm{AB}}$ diblocks must pass through a region of spheres and cylinders composed of the minority B block mixed with the $\mathrm{C}$ homopolymer before transitioning to lamellae by continuity of the effective parameter mean field phase diagram. A schematic diagram of this process is shown in Figure S8. In the real system, fluctuation effects likely made such phases unstable and thus not observed at low values of $f_{\mathrm{C}}$. 




Figure S8: Diagram showing how by continuity the addition of PVPH to the PS- $b$-PMMA must go through a phase of PMMA rich spheres and PMMA rich cylinders before lamellae in the mean field limit of the simulations. The upper left corner shows a schematic of the mean field phase diagram of the effective $\chi N$ versus effective minority component volume fraction $f_{\text {eff }}$ with the canonical phases such as spheres (S), cylinders $(\mathrm{C})$, gyroid $(\mathrm{G})$, lamaellae $(\mathrm{L})$, and disordered (Dis) labelled (note more complex phases with narrow existence regions are omitted for clarity). The lower right corner shows a zoom-in view of the relevant region of the phase diagram of interest for the effect adding PVPH homopolymer has on the system. The orange and purple arrow lines show potential path for $\chi_{\text {eff }} N$ and $f_{\text {eff }}$ to traverse as $f_{\mathrm{C}}$ increases for the $N_{\mathrm{AB}}=183$ and 196 diblocks, respectively. The dots at the beginning of the arrows represent $f_{\mathrm{C}}=0$. In both cases, the polymer must pass through sphere and cylinder phases before entering the lamellae regime. 


\section{S5 Chain End Distribution Comparison:}

To better understand why $a_{\mathrm{j}}$ continued to increase with $f_{\mathrm{C}}$ in the simulations and not in the experiment, plots of the chain end distribution $\hat{q}(\hat{x})$ for each species are shown in Figure S9 along with the density of the corresponding species, where $\hat{x}$ is the normalized position such that $\hat{x}=x / L_{0}$ and $\hat{q}$ is the chain end propagator normalized to have a maximum value of 1 such that $\hat{q}_{Z}(\hat{x})=q_{Z}\left(x, s=N_{\mathrm{S}, Z}\right) / \max \left(q_{Z}\left(x, s=N_{\mathrm{S}, Z}\right)\right)$. These distribution plots are for a representative $3 \mathrm{D}$ lamellae results taken from $\chi_{\mathrm{BC}}=-0.05, N_{\mathrm{AB}}=196$, and $f_{\mathrm{C}}=0.37$. The results were found to be invariant along the plane of the lamellae (in the $y$ and $z$ directions), thus only plots along the $x$ direction are shown. From the plots, the chain ends are seen to concentrate at the center of the respective domains for all species. The density distribution peaks towards the center for the PS and PVPH domains, while the PMMA domain has two peaks located between the PS and PMMA interface and central PVPH domain region. These observations show that the chains are strongly stretched in the simulation, but cannot stretch out of the plane of the lamellae because of the Gaussian coil model used in the simulations. A wormlike chain model may be able to perform such anisotropic stretching, thus future work should examine such a model to see if the chains do indeed stretching perpendicular to the interface to accommodate the increasing $a_{\mathrm{j}}$. Seeing that the hydrogen bonding of the PVPH and PMMA would lead to a more rigid structure, a wormlike chain model might capture such an effect better. 


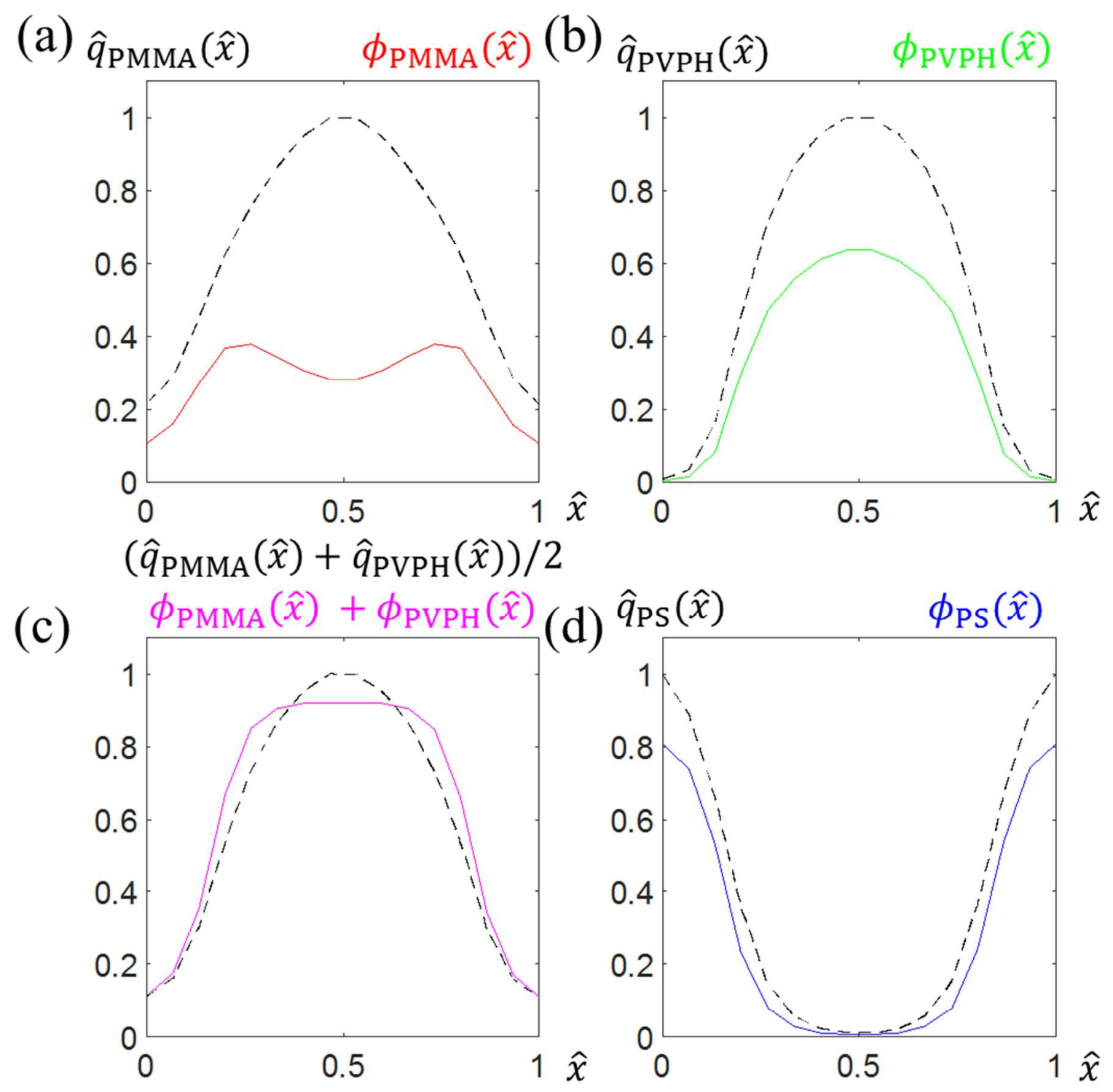

Figure S9: Plots of the normalized chain end distributions $\hat{q}$ as a function of normalized position $\hat{x}$ for the respective species along with density profiles for a 3D representative lamellae. $x$ is the direction parallel to the normal of the lamellae interface. Black dashed lines are the chain end distributions and colored solid lines are the densities $\phi$. (a) Plot for the PMMA. (b) Plot for the PVPH. (c) Plot for the sum of the PMMA and PVPH domain. (d) Plot for the PS. 
S6 Additional SANS Data:
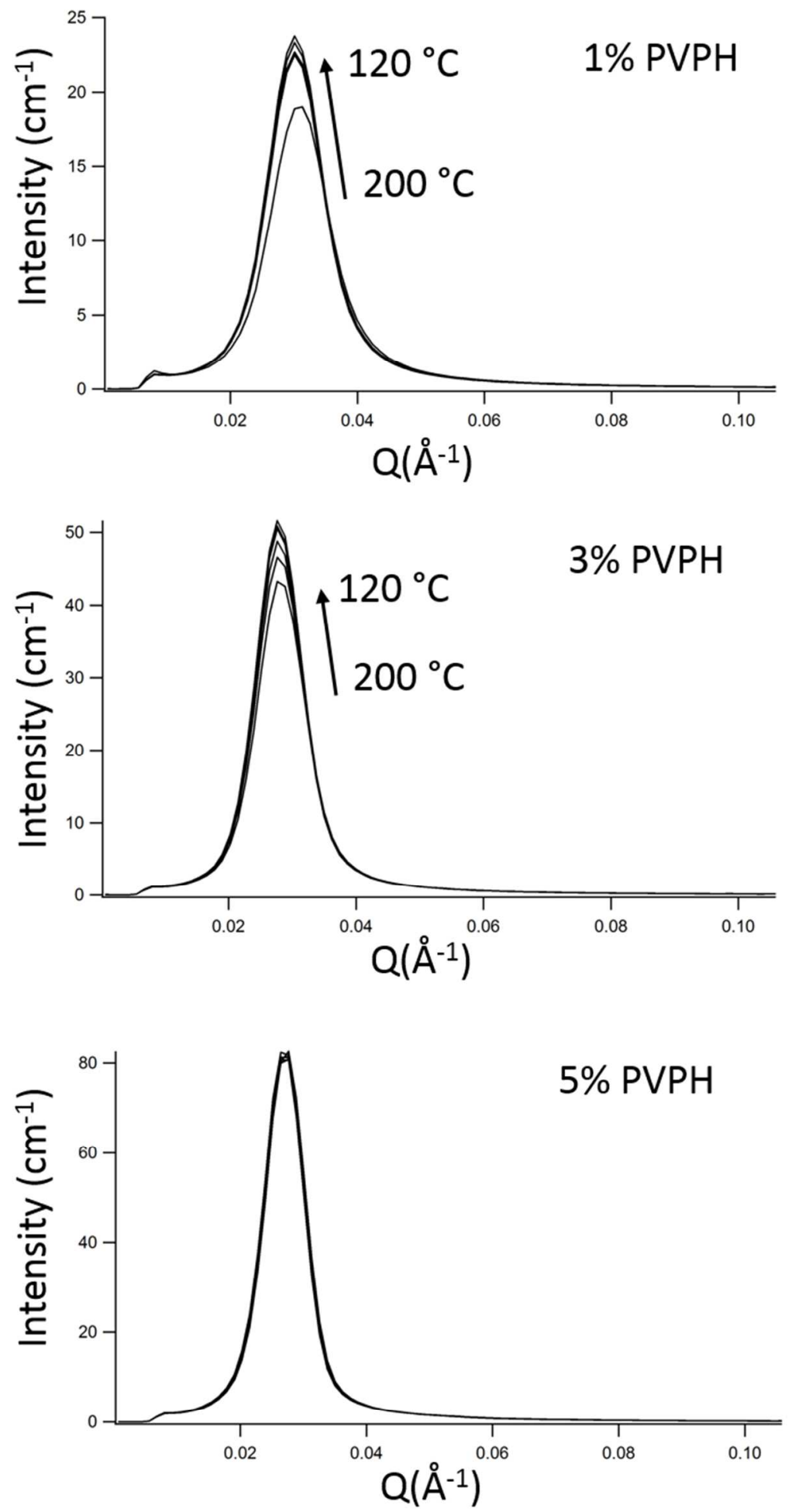

Figure S10: SANS measurements on $\mathrm{PVPH}_{7} / \mathrm{dPS}_{11}-b-\mathrm{PMMA}_{8}$ as a function of temperature for A) $\Phi_{H}=0.01$, B) $\Phi_{\mathrm{H}}=0.03$, C) $\Phi_{\mathrm{H}}=0.05$. 


\section{List of Abbreviations and Symbols}

Abbreviations/Acronyms

A: Polymer species in simulations corresponding to PS in experiments

B: Polymer species in simulations corresponding to PMMA in experiments

C: Polymer species in simulations corresponding to PVPH in experiments

BCP: Block copolymer; DSA: Directed self-assembly

ODT: Order-disorder transition; OOT: Order-order transition

PDI: Polydispersity index;

P2VP: Poly(2-vinylpyridine) PAA: Poly(acrylic acid) PEO: Poly(ethylene oxide)

PGMEA: Propyl glycol methyl ether acetate PI: Polyisoprene PMMA: Poly(methyl methacrylate)

PPO: Poly(propylene oxide) PS: Polystyrene PVP: Polyvinylpyrolidone PVPH: Poly(vinyl phenol)

SANS: Small angle neutron scattering; SAXS: Small angle X-ray scattering

SCFT: Self-consistent field theory

Symbols/Variables

$\chi$ Flory-Huggins interaction parameter

$\eta$ Ratio of homopolymer degree of polymerization to hydrogen bonding block degree of polymerization

$N_{\mathrm{B}}^{\mathrm{BCP}}$ Degree of polymerization of B species in BCP

$N_{\mathrm{B}}^{\mathrm{H}}$ Degree of polymerization of B species homopolymer

$f_{\mathrm{H}}$ Volume fraction of homopolymer added (also $f_{\mathrm{B}}^{\mathrm{H}}$ )

$\Phi_{\mathrm{H}}$ Mass fraction of homopolymer added

$\Phi_{\mathrm{c}}$ Critical concentration where macrophase separation occurs

$a_{\mathrm{j}}$ Area per junction under a given blending condition

$a_{\mathrm{j} 0}$ Area per junction without homopolymer added

$L_{0}$ System natural periodicity without homopolymer added

$L$ System periodicity under a given blending condition

$L_{\mathrm{PS}}$ Length of a PS domain; $L_{\mathrm{Bl}}$ Length of PMMA/PVPH blended domain

$\lambda$ Wavelength of scattered neutron or X-ray beam

$q$ Reciprocal space variable 
$\theta$ Incident angle of beam with sample

$Z$ Total partition function in SCFT simulations

$H$ Hamiltonian functional in SCFT simulations

$k$ Boltzmann constant; $T$ Temperature

$\rho$ Set of density fields in SCFT simulations $\rho \equiv\left\{\rho_{\mathrm{A}}, \rho_{\mathrm{B}}, \rho_{\mathrm{C}}\right\}$

$\Omega$ Set of chemical potential fields in SCFT simulations $\Omega \equiv\left\{\Omega_{\mathrm{A}}, \Omega_{\mathrm{B}}, \Omega_{\mathrm{C}}\right\}$

$D$ Functional integral differential operator (designates which functional to integrate over)

$\overrightarrow{\mathbf{r}}$ Position vector corresponding to real space coordinates $x, y$, and $z$

$N_{x}, N_{y}$, and $N_{z}$ Grid point size in corresponding real space coordinate direction

$\rho^{*}$ and $\Omega^{*}$ Field solutions that satisfy SCFT saddle point condition

$F$ Free energy; $\Delta F$ Free energy difference from reference disordered homogeneous value

$\phi$ Set of normalized density fields in SCFT simulations $\phi \equiv\left\{\phi_{\mathrm{A}}, \phi_{\mathrm{B}}, \phi_{\mathrm{C}}\right\}$

$\rho_{0}$ Average monomer density

$G$ Ginzburg parameter that scales energy in Hamiltonian with system size

$R_{\mathrm{g}}$ Radius of gyration of the $\mathrm{AB}$ block

$N$ Degree of polymerization of the AB block

$\chi_{X Y}$ Flory-Huggins interaction parameter between species $X$ and $Y$ (can be A, B, or C)

$N_{Z}$ Degree of polymerization of chain $Z ; R_{\mathrm{g}, Z}$ Radius of gyration of chain $Z$ (chain can be $\mathrm{AB}$

$\mathrm{BCP}$, B homopolymer, or $\mathrm{C}$ homopolymer)

$f_{X}$ Volume fraction of species or chain $X$

$\Omega_{\mathrm{P}}$ Pressure field used to enforce incompressibility in the system

$\phi_{+}$Sum of all species normalized density (should equal 1 everywhere for true incompressibility)

$V$ Total volume of the system

$Q_{Z}$ Degree of polymerization of chain $Z$

$q_{Z}$ Chain propagator function of chain $Z$

$s$ Chain position variable; $N_{\mathrm{S}, Z}$ Statistical chain segment length of chain $Z$

$q_{Z}^{\dagger}$ Backward chain propagator function of chain $Z$

$\lambda_{j}$ Relaxation constant used in field relaxation scheme 
$j$ Index used in relaxation scheme

$W_{j}$ Species demarcation based on index $j$

$X, Y$, and $Z$ Species variables in relaxation scheme that correspond to $\mathrm{A}, \mathrm{B}$, and $\mathrm{C}$ in different permutations

$t$ Time step in simulation; $J$ Iteration index

$k_{j}$ and $m_{j}$ Subindices based on index $j$

$\xi$ Gaussian distributed random noise

$\kappa$ Compressibility constant

$\mathrm{H}$ Superscript to distinguish homopolymer $\mathrm{B}$ from $\mathrm{B}$ in the $\mathrm{AB}$ diblock

$\alpha$ Ratio of the $\mathrm{C}$ homopolymer block to the $\mathrm{AB}$ diblock degree of polymerization

$\Lambda$ Length scale assigned to the simulation grid

$b$ Monomer Kuhn segment length

$\Delta F_{\text {fit }}$ Fit free energy using strain dominated relationship

$\Gamma$ Quadratic fitting constant in $\Delta F_{\text {fit }}$

$\beta$ Lorentzian fitting constant in $\Delta F_{\text {fit }}$

$\epsilon$ Shift fitting constant in $\Delta F_{\text {fit }}$

a.u. Arbitrary units assigned in simulation length scale such that 4 statistical monomers is 1 a.u.

$L / L_{0}$ Ratio of system period under a given blending condition to bulk copolymer period

$M_{\mathrm{PS}}$ Molecular mass of PS

$N_{\text {Av }}$ Avagadro's constant

$\rho_{\text {Ps }}$ Density of PS

$L_{\text {Sat }}$ Saturation $L$ value in extrapolation fits

$\zeta$ Growth constant in extrapolation fits

$\chi_{\text {eff }}$ Effective $\mathrm{AB} \chi$ parameter from homopolymer incorporation

$f_{\text {eff }}$ Effective fraction of minority component in system as a function added homopolymer

$\hat{q}$ Chain end distribution function

$\hat{x}$ Normalized position 


\section{SI References:}

(1) Matsen, M. W. Macromolecules 1995, 28, 5765-5773.

(2) Bates, F. S.; Fredrickson, G. H. Annu. Rev. Phys. Chem. 1990, 41, 525-557.

(3) Hansen, N.; Ostermeier, a. Evol. Comput. 2001, 9, 159-195.

(4) Hansen, N.; Müller, S.; Koumoutsakos, P. Evol. Comput. 2003, 11, 1-18.

(5) Hansen, N.; Kern, S. Proc. 8th Int. Conf. Parallel Probl. Solving from Nat. - PPSN VIII 2004, 3242/2004, 282-291. 